\title{
GRB 090227B: A FIRST EXAMPLE OF A GENUINE SHORT GRB
}

\author{
M. MUCCINO*, C. L. BIANCO ${ }^{\dagger}$, L. IZZO ${ }^{\ddagger}$, A. V. PENACCHIONI ${ }^{\S}$ and R. RUFFINI \\ Dip. di Fisica, Sapienza Università di Roma \\ Piazzale Aldo Moro 5, I-00185 Rome, Italy \\ ICRANet, Piazza della Repubblica 10, I-65122 Pescara, Italy \\ Universite de Nice Sophia Antipolis, Nice, CEDEX 2 \\ Grand Chateau Parc Valrose, France \\ *marco.muccino@icra.it \\ †bianco@icra.it \\ $\ddagger$ luca.izzo@icra.it \\ §ana.penacchioni@icra.it \\ Iruffini@icra.it
}

\begin{abstract}
In the context of the Fireshell scenario Gamma-Ray Bursts (GRBs) find a natural explanation in the values of three main parameters: the energy of the emitting $e^{ \pm}$plasma $E_{e^{ \pm}}^{t o t}$, the baryon load $B$, and the density of the Circum-Burst Medium (CBM) $n_{C B M}$. For $B \lesssim 10^{-5}$, the GRB consists of a spike-like emission without any afterglow: a genuine short burst. GRB 090227B is the first recognized genuine short GRB. Its analysis reveals that the transparency emission is not a pure thermal process, owing to a non-gradual transition between the optically thick and the optically thin phases. Within our theory we have recovered the original thermal spectrum of the $e^{ \pm}$plasma, namely the effective Black body (BB); we indeed have determined the parameters of the Fireshell model, including the redshift. Setting a baryon load $B=10^{-6}$, we obtain the effective temperature $k \mathcal{T}=(719.3 \pm 48.5) \mathrm{keV}$, the Lorentz factor at transparency $\Gamma=12852$, and the total energy $E_{e^{ \pm}}^{t o t}=(1.13 \pm 0.12) \times 10^{54} \mathrm{erg}$. The estimated redshift is $z=4.07 \pm 0.36$.
\end{abstract}

Keywords: Gamma-ray burst: general; gamma-ray burst: individual: GRB 090227B.

PACS numbers: $98.70 . \mathrm{Rz}$

\section{Introduction}

Current and past missions dedicated to the observation of GRBs, as the Fermi Gamma-ray Burst Monitor (GBM), ${ }^{1}$ BATSE, ${ }^{2}$ and others, have evidenced a temporal distribution in the duration of the prompt emission of GRBs. ${ }^{3}$ Defining the $T_{90}$ as the time interval over which the $90 \%$ of the total background-subtracted counts are observed, long and short GRBs were classified as being longer or shorter than $T_{90}=2 \mathrm{~s}$. This classification strictly depends on the instrumental features and, after the discovery of the afterglow by Beppo-SAX, ${ }^{4}$ it has been questioned by the existence of a class of GRBs with hybrid behavior between short and long. ${ }^{5}$ This "disguised" short class ${ }^{6}$ finds a natural explanation within the Fireshell model. ${ }^{7}$ 
Within our theory, the existence of a class of "genuine" short GRBs is predicted. The characteristic duration of these bursts is expected to be of the order of $10^{-2} \mathrm{~s}$, and they have no associated afterglow. We present the analysis of the first identified genuine short burst, GRB 090227B.

The paper is organized as follows: in Sec. 2 we present the observations of GRB 090227B and the data analysis; in Sec. 3 we determine all the quantities of GRB 090227B within the Fireshell scenario, including its redshift, and we prove that it cannot be a disguised short GRBs; finally, we present our conclusions.

\section{Observations and Data Analysis of GRB 090227B}

At 18:31:01.41 UT on $27^{\text {th }}$ February 2009, the Fermi GBM detector ${ }^{8}$ triggered and located the short and bright burst, GRB 090227B (trigger 257452263/090227772). The on-ground calculated location, using the GBM trigger data, is $(\mathrm{RA}, \mathrm{Dec})(\mathrm{J} 2000)=\left(11^{h} 48^{m} 36^{s}, 32^{\circ} 10^{\prime} 12^{\prime \prime}\right)$, with an uncertainty of $1.77^{\circ}$ (statistical only). The burst was also located by $\mathrm{IPN}^{9}$ and detected by KonusWind, ${ }^{10}$ showing a single pulse with $T_{90} \sim 0.2 \mathrm{~s}(20 \mathrm{keV}-10 \mathrm{MeV})$. No X rays and optical observations were reported, thus the redshift of the source is unknown.

The light curves from the NaI-n2 (8-900 keV) (Fig. 1, left panel) and the BGOb0 $(250 \mathrm{keV}-40 \mathrm{MeV})$ detectors show one bright spike having duration $T_{90}=0.382$ s. Time integrated spectral analysis results are summarized in Tab. 1. The best fit is a combination of Black Body (BB) and Band ${ }^{11}$ spectral models, with the thermal component superimposed by the non-thermal one (Fig. 1, right panel).
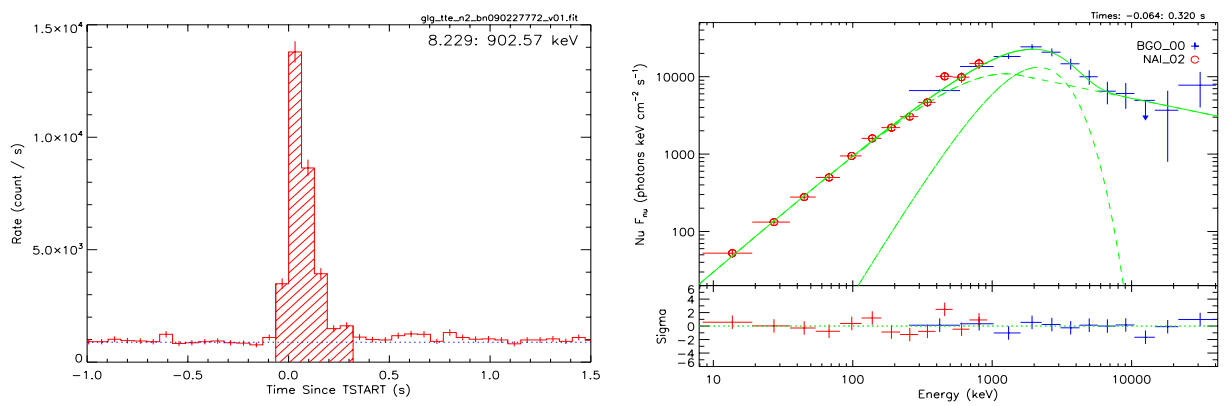

Fig. 1. The NaI-n2 light curve (left panel) and the NaI-n2+BGO-b0 $\nu F_{\nu}$ spectrum (right panel) of GRB 090227B; the best fit shows a BB component, superimposed by a Band spectrum.

Table 1. The time integrated spectral analysis performed using BB+Band model of GRB 090227B in the fireshell scenario.

\begin{tabular}{ccccccc}
\hline $\begin{array}{c}\mathrm{kT} \\
{[\mathrm{keV}]}\end{array}$ & $\alpha$ & $\beta$ & $\begin{array}{c}E_{p} \\
{[\mathrm{keV}]}\end{array}$ & $\begin{array}{c}F_{\text {tot }} / 10^{-5} \\
{\left[\mathrm{erg} /\left(\mathrm{cm}^{2} \mathrm{~s}\right)\right]}\end{array}$ & $\begin{array}{c}F_{B B} / 10^{-5} \\
{\left[\mathrm{erg} /\left(\mathrm{cm}^{2} \mathrm{~s}\right)\right]}\end{array}$ & $\begin{array}{c}\mathrm{C}-\mathrm{STAT} / \\
\mathrm{DOF}\end{array}$ \\
\hline $545.7 \pm 36.8$ & $-0.46 \pm 0.04$ & $-2.37 \pm 0.21$ & $1252 \pm 280$ & $8.67 \pm 0.28$ & $2.87 \pm 1.35$ & $279.6 / 238$ \\
\hline
\end{tabular}




\section{Analysis of GRB 090227B within the Fireshell Model}

In the Fireshell model, ${ }^{7}$ all GRBs originate from the vacuum polarization process occurring around a black hole. The optically thick formed $e^{ \pm}$plasma, having total energy $E_{e^{ \pm}}^{\text {tot }}$, is in thermal equilibrium ${ }^{12}$ and it is confined in an expanding shell, called fireshell, which self-accelerates up to ultra relativistic velocities, and engulfs the baryonic remnant, which thermalizes with the pairs. ${ }^{13}$ The amount of baryons is described by the baryon load $B=M_{B} c^{2} / E_{e^{ \pm}}^{\text {tot}}$. The canonical GRBs is composed of the emission due to the transparency of the fireshell, the Proper-GRB (P-GRB), and the extended afterglow due to the collisions between the accelerated baryons and the CBM, having density $n_{C B M}$. According to this theory, when the baryon load is $3.0 \times 10^{-4} \lesssim B \leq 10^{-2}$ and the CBM density is $\left\langle n_{C B M}\right\rangle \approx 1$ particles $/ \mathrm{cm}^{3}$, the extended afterglow emission is predominant, giving rise to the "long" GRBs (see Fig. 2). For $\left\langle n_{C B M}\right\rangle \approx 10^{-3}$ particles $/ \mathrm{cm}^{3}$, typical of galactic halos, the extended afterglow results in a weaker emission, giving rise to the "disguised short" GRBs.

There is a third theoretically predicted class of GRBs, the so-called genuine short GRBs. ${ }^{14}$ They occur for $B<10^{-5}$, with typical intrinsic durations $\sim 10^{-2} \mathrm{~s}$, and their spectral energy distribution (SED) is expected to be thermal. In addition they have no observed optical afterglow, and consequently no redshift determination.
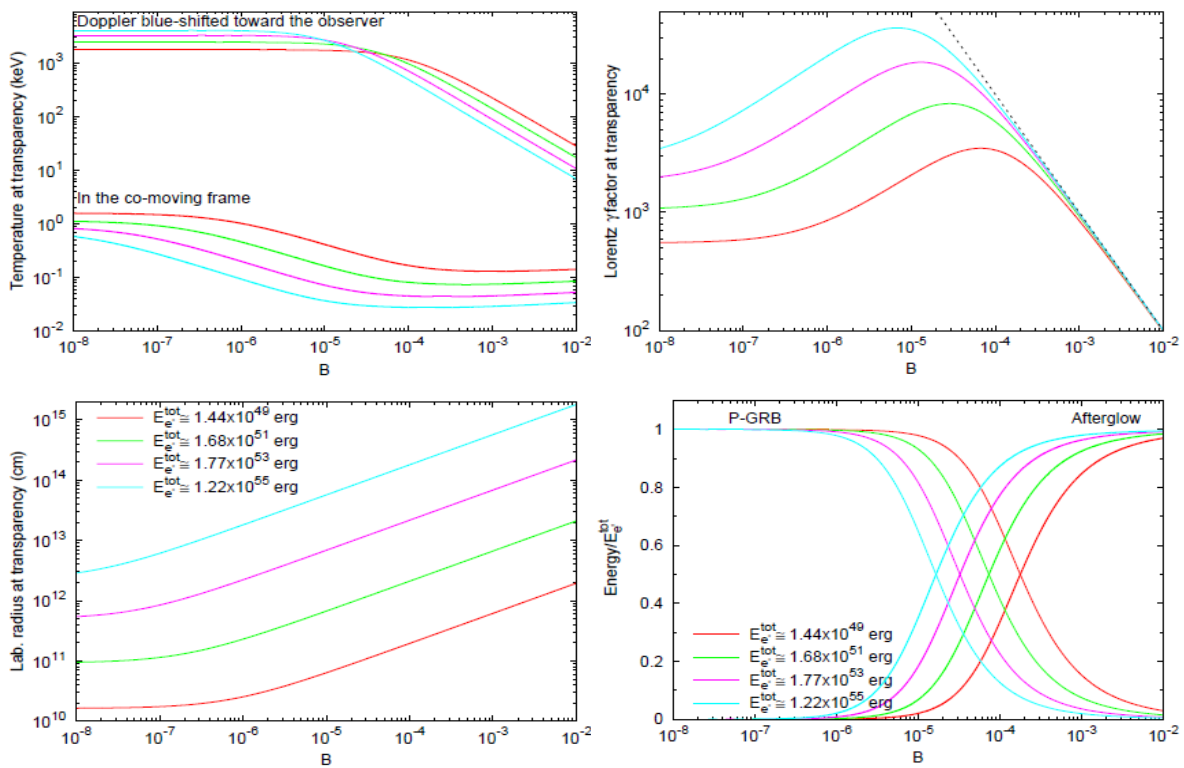

Fig. 2. The main quantities of the Fireshell model at the transparency for selected values of $E_{e^{ \pm}}^{\text {tot }}$ : the laboratory radius, the co-moving and blue-shifted toward the observer temperatures of the plasma, the Lorentz factor, and the fraction of the energy radiated in the P-GRB and in the extended afterglow as functions of $B$. 
Table 2. The effective BB and the Fireshell model parameters of GRB 090227B. The errors follow directly from the observation, while the fixed values from the theory.

\begin{tabular}{cccccccc}
\hline$\kappa$ & $\begin{array}{c}k \mathcal{T} \\
{[\mathrm{keV}]}\end{array}$ & $\begin{array}{c}E_{e^{ \pm}}^{\text {tot }} \\
{[\mathrm{erg}]}\end{array}$ & $B$ & $\Gamma_{\text {tr }}$ & $\begin{array}{c}r_{\text {tr }} \\
{[\mathrm{cm}]}\end{array}$ & $\begin{array}{c}k T_{\text {blue }} \\
{[\mathrm{keV}]}\end{array}$ & $z$ \\
\hline 2.02 & $719.3 \pm 48.5$ & $(1.13 \pm 0.12) \times 10^{54}$ & $10^{-6}$ & 12852 & $5.54 \times 10^{12}$ & 3649 & $4.07 \pm 0.36$ \\
\hline
\end{tabular}

GRB 090227B is the first identified genuine short burst. This source offers the opportunity to investigate a pure $e^{ \pm}$plasma transparency. Its spectrum shows a clear BB component, superimposed by a Band one (see Fig. 1). We assumed that the non-thermal component originates in a gradual transition between the optically thick and the optically thin phases, instead of a sudden transition adopted in Fig. 2. We have consequently estimated the original thermal spectrum of the $e^{ \pm}$plasma, in other words the effective $\mathrm{BB}$ spectrum, simply setting the total observed flux $F_{\text {tot }}$ equal to the flux of the effective thermal component $\mathcal{F}_{B B}$, thus

$$
\mathcal{F}_{B B}=F_{B B}+F_{\text {extra }}=F_{B B}(1+\kappa),
$$

where $\kappa \equiv F_{\text {extra }} / F_{B B}$. Using the definition of the flux of the BB, we obtain a relation between the temperature of the effective $\mathrm{BB} k \mathcal{T}$ and the observed one $k T$

$$
k \mathcal{T}=k T(1+\kappa)^{\frac{1}{4}} .
$$

By setting $B=10^{-6}$ and varying $E_{e^{ \pm}}^{t o t}$, we have computed the Doppler blueshifted toward the observer temperature of the plasma at transparency, $k T_{\text {blue }}$, and then, by the ratio $k T_{\text {blue }} / k \mathcal{T}=(1+z)$, the redshift $z$. The correct value of $z$ is the one for which the isotropic energy $E_{i s o}=4 \pi d_{l}^{2} F_{t o t} T_{90} /(1+z)\left(d_{l}\right.$ is the luminosity distance) is equal to $E_{e^{ \pm}}^{\text {tot }}$. This condition occurs for $z=4.07 \pm 0.36$, that means for $E_{\text {iso }}=(1.13 \pm 0.12) \times 10^{54} \mathrm{erg}$. The Fireshell model quantities, as well as the redshift, and the effective BB parameters, are summarized in the Tab.2.

Finally we reject the possibility that GRB 090227B is a disguised short burst with an extended afterglow below the instrumental threshold. We have set $E_{e^{ \pm}}^{t o t}=$ $2.26 \times 10^{54} \mathrm{erg}$ and $B=2.05 \times 10^{-5}$, corresponding to the condition $E_{P-G R B}=$ $50 \% E_{e^{ \pm}}^{t o t}$ that marks the division between the genuine short and disguised short/long GRBs (Fig. 2). We have simulated the photon flux light curve of the virtual extended afterglow in the energy range 50-300 keV which maximize the sensitivity of the GBM detectors and we have compared it with the corresponding threshold ${ }^{\mathrm{a}}, \phi_{t h r} \lesssim 0.5$ photons $/\left(\mathrm{cm}^{2} \mathrm{~s}\right)$ (Fig. 3, left panel). Similarly we have simulated the energy flux light curve in the energy range $0.3-10 \mathrm{keV}$ of X-Ray Telescope (XRT), on-board the Swift satellite $^{15}$ (Fig. 3, right panel). The sensitivity limit of XRT is $F_{X R T} \approx 2 \times 10^{-14}$ $\mathrm{erg} /\left(\mathrm{cm}^{2} \mathrm{~s}\right)$ within $10^{4} \mathrm{~s}$ of observation in the same energy range ${ }^{\mathrm{b}}$.

\footnotetext{
${ }^{a}$ http://fermi.gsfc.nasa.gov/ssc/data/analysis/documentation/Cicerone/Cicerone_ Introduction/ GBM_overview.html

bhttp://www.swift.psu.edu/xrt/techDescription.html
} 

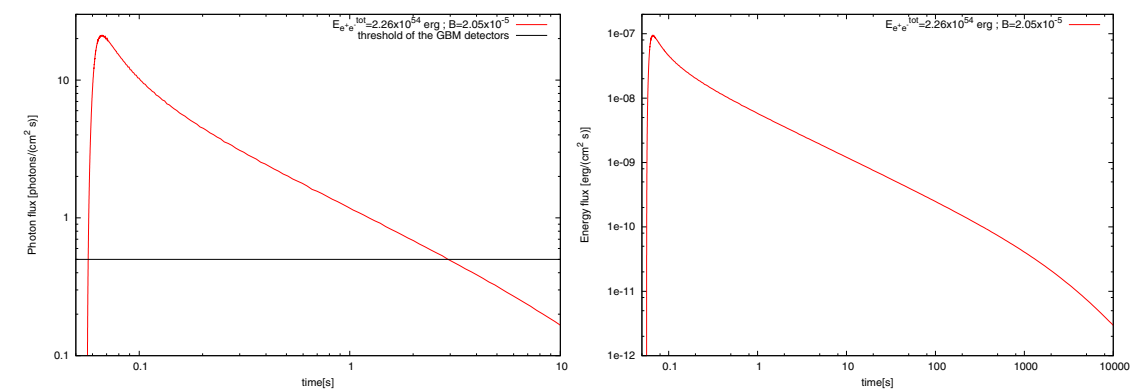

Fig. 3. The simulated light curves of the virtual extended afterglow: on the left, the photon flux light curve in the energy range 50-300 keV compared with the threshold of the GBM detectors, and on the right, the energy flux light curve in the energy range $0.3-10 \mathrm{keV}$.

We can conclude that if any extended afterglow emission had occurred, it could have been detected. As a result GRB 090227B is a genuine short burst.

\section{Conclusions}

Within the Fireshell scenario we have proved that GRB 090227B is the first genuine short burst. It offers a full comprehension of a pure $e^{ \pm}$plasma transparency that clearly shows an expected thermal signature, but superimposed by a nonthermal component originating in the gradual transparency condition, instead of the sudden one assumed in Fig. 2. Consequently, we have recovered the original thermal spectrum of the plasma, indeed the effective temperature of the plasma, $k \mathcal{T}=(719.3 \pm 48.5) \mathrm{keV}$, and then the total energy emitted, $E_{e^{ \pm}}^{t o t}=(1.13 \pm 0.12) \times 10^{54}$ $\mathrm{erg}$, the Lorentz factor, $\Gamma_{t r}=12852$, and finally the redshift, $z=4.07 \pm 0.36$.

\section{References}

1. C. Meegan, G. Lichti, P. N. Bhat, et al., ApJ, 702, 791 (2009).

2. C. A. Meegan, G. J. Fishman, R. B. Wilson, et al., Nature, 355, 143 (1992).

3. R. W. Klebesadel, The durations of gamma-ray bursts, eds. C. Ho, R. I. Epstein, R. I. and E. E. Fenimore, p. 161168 (1992).

4. E. Costa, F. Frontera, J. Heise, et al., Nature, 387, 783 (1997).

5. J. P. Norris and J. T. Bonnell, ApJ, 643, 266 (2006).

6. M. G. Bernardini, C. L. Bianco, L. Caito, et al., A\&4A, 474, L13 (2007).

7. R. Ruffini, C. L. Bianco, F. Fraschetti, S.-S. Xue and P. Chardonnet, ApJ, 555, L113 (2001).

8. S. Guiriec, GRB Coordinates Network, 8921, p. 1 (2009).

9. S. Golenetskii, R. Aptekar, E. Mazets, et al., GRB Coordinates Network, 8925, p. 1 (2009).

10. S. Golenetskii, R. Aptekar, E. Mazets, et al., GRB Coordinates Network, 8926, p. 1 (2009).

11. D. Band, J. Matteson, L. Ford, et al., ApJ, 413, 281 (1993). 
12. A. G. Aksenov, R. Ruffini and G. V. Vereshchagin, Physical Review Letters, 99, 125003 (2007).

13. R. Ruffini, J. D. Salmonson, J. R. Wilson and S.-S. Xue, A\&AA, 359, 855 (2000).

14. R. Ruffini, C. L. Bianco, P. Chardonnet, F. Fraschetti and S.-S. Xue, ApJ, 581, L19 (2002).

15. N. Gehrels, C. L. Sarazin, P. T. OBrien, et al., Nature, 437, 851 (2005). 